\title{
硅酸钙纳米线复合电纺丝支架的制备及离子释放研究
}

\author{
包峰 1,2 , 常 江 1,2 \\ (1. 中国科学院 上海硅酸盐研究所, 高性能陶瓷与超微结构国家重点实验室, 上海 200050; 2. 中国科学院大学 材料与光 \\ 电研究中心, 北京 100049)
}

摘 要: 电纺丝支架已被广泛用于组织工程领域, 其中硅酸钻等生物活性陶瓷复合的电纺丝支架, 在应用中展现出 了优异的生物活性。硅酸钙复合电纺丝支架中硅酸钲降解释放的硅酸根离子 $\left(\mathrm{SiO}_{3}{ }^{2-}\right)$ 已被证实具有促进成血管性 能, 但其有效活性离子浓度范围比较窄, 仅在 $0.79 \sim 1.8 \mu \mathrm{g} / \mathrm{mL}$ 之间。因此精确控制组织工程材料的离子释放浓度, 使材料释放的离子能较长时间保持在有效活性浓度范围, 对于组织工程应用具有重要意义。本研究通过调节电纺 丝孔径大小及硅酸钻纳米线的不同复合方式, 制备了多种硅酸钻复合电纺丝纤维支架, 并比较了其在体外环境下 的离子释放模式及对人脐静脉内皮细胞的增殖促进作用。实验结果表明, 混纺及同时电喷-电纺复合方式的小孔径 硅酸钻复合电纺支架由于高分子的疏水作用和小孔径结构对离子扩散的阻碍, 可以实现离子缓释。通过体外细胞 实验发现, 具有离子缓释效果的支架可以更好地促进人脐静脉内皮细胞的增殖, 说明通过调控支架离子缓释, 可 以有效调控其生物活性, 获得最佳组织工程应用效果。

关 键 词: 硅酸钙; 静电纺丝; 可控释放; 降解; 静电喷雾

中图分类号: R318 文献标识码: A

\section{Calcium Silicate Nanowires Based Composite Electrospun Scaffolds: Prepara- tion, Ion Release and Cytocompatibility}

\author{
BAO Feng ${ }^{1,2}$, CHANG Jiang ${ }^{1,2}$
}

(1. State Key Lab of High Performance Ceramics and Superfine Microstructure, Shanghai Institute of Ceramics, Chinese Academy of Sciences, Shanghai 200050, China; 2. Center of Materials Science and Optoelectronics Engineering, University of Chinese Academy of Sciences, Beijing 100049, China)

\begin{abstract}
Electrospun scaffolds have been widely used in tissue engineering, especially bioceramics such as calcium silicate (CSH) composite electrospun scaffolds have shown excellent bioactivity by releasing $\mathrm{SiO}_{3}{ }^{2-}$ ions during the degradation of calcium silicate in composite electrospun scaffolds, which are bioactive in stimulating angiogenesis. However, the effective ion concentration is in a narrow range from 0.79 to $1.8 \mu \mathrm{g} / \mathrm{mL}$. Therefore, it is of great significance for tissue engineering applications to accurately control the ion release concentration of the calcium silicate composite scaffolds, so that the ions released from materials can remain in the effective concentration range for a long time. In this study, we prepared a variety of scaffolds with calcium silicate composite electrospun by adjusting pore size of electrospun scaffolds and controlling the location of calcium silicate nanowires inside the scaffolds, and compared ions release behavior and bioactivity in promoting proliferation of human umbilical vein endothelial cells in vitro. The results showed that, due to the hydrophobicity of polymers and limited diffusion of small pore size, electrospun scaffolds with small pore size by mixed-electrospinning or electrospinning-electrospraying calcium silicate composite displayed slow-release behavior of $\mathrm{SiO}_{3}{ }^{2-}$ ions. In vitro cell experiments showed that the electrospun scaffolds with slow ions release promoted
\end{abstract}

收稿日期：2021-01-28; 收到修改稿日期：2021-03-24

基金项目：国家重点研发计划（2016YFC1100201） National Key Research and Development Program of China (2016YFC1100201)

作者简介：包 峰（1991-)，男，博士研究生. E-mail: baofeng@ student.sic.ac.cn

BAO Feng (1991-), male, PhD candidate. E-mail: baofeng@student.sic.ac.cn

通信作者: 常 江, 研究员. E-mail: jchang@mail.sic.ac.cn

CHANG Jiang, professor. E-mail: jchang@mail.sic.ac.cn 
the proliferation of human umbilical vein endothelial cells, indicating that the bioactivity of the composite scaffolds could be regulated by adjusting ions release behavior to obtain optimal bioactivity for tissue engineering applications.

Key words: calcium silicate; electrospun; controllable release; degradation; electrospray

静电纺丝支架因具有类细胞外基质的三维结构、 大比表面积、高孔隙率 ${ }^{[1-3]}$, 被广泛应用于血管 ${ }^{[4-5] 、}$

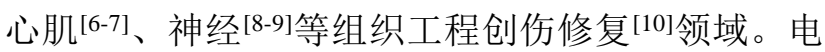
纺丝支架经过多年的研究发展, 通过在其中引入活 性药物 ${ }^{[11]}$ 和活性陶瓷等 ${ }^{[12]}$ 赋予了电纺丝支架粘附、 增殖、迁移、分化等生物活性。

硅酸钻等生物活性陶瓷分解释放的 $\mathrm{SiO}_{3}{ }^{2-}$ ，不 仅被证实具有促进骨 ${ }^{[13]}$ 、牙齿 ${ }^{[14]}$ 等硬组织再生的作 用，近年来陆续发现 $\mathrm{SiO}_{3}{ }^{2-}$ 还具有促进血管 ${ }^{[15]}$ 、脂

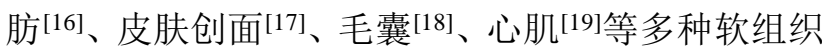
修复的生物活性。前期研究中，硅酸钻等生物活性 陶瓷通过混纺 ${ }^{[19]}$ 或者喷涂 ${ }^{[20]}$ 的方式与电纺丝进行 复合, 促进了组织的修复。但是, 不同的组织修复 所需的活性 $\mathrm{SiO}_{3}{ }^{2-}$ 浓度范围各有差异 ${ }^{[21]}$, 以促进成 血管性能为例, 据 $\mathrm{Li}$ 等 ${ }^{[22]}$ 报道, $\mathrm{SiO}_{3}{ }^{2-}$ 有效促进成 血管的浓度范围为 $0.79 \sim 1.8 \mu \mathrm{g} / \mathrm{mL}$, 有效浓度范围较 窄, 这对于材料中 $\mathrm{SiO}_{3}{ }^{2-}$ 的释放行为提出了要求。虽 然前述工作报道的硅酸钻等生物活性陶瓷复合电纺 丝支架中释放的 $\mathrm{SiO}_{3}$ 2-具有促进软组织修复的作用, 但并未关注材料中 $\mathrm{SiO}_{3}{ }^{2-}$ 的释放行为对于软组织修 复效果的影响。因此, 精确控制组织工程材料中硅 酸盐陶瓷降解产生的 $\mathrm{SiO}_{3}{ }^{2-}$ 的释放行为具有重要意 义。

前期的研究 ${ }^{23-25]}$ 表明, 孔径的大小对材料内部 的药物释放有重要影响, 因此本研究提出的第一个 假设是，通过调节电纺丝的孔径结构可以有效调控 生物活性离子的释放行为。此外, 医用电纺丝支架 常用的高分子主要有聚己内酯(PCL)、聚乳酸(PLA) 等疏水性高分子，有文献 ${ }^{[26]}$ 报道这些疏水性高分子 的疏水作用将阻碍内部药物释放, 因此本研究提出 第二个假设是，硅酸钻在复合材料体系内的分布位 置不同, 如纤维丝内部、纤维表面均匀分布、集中 分布于支架外表面、“三明治” 夹心层等, 将会导致 复合材料中硅酸钙受到的高分子疏水作用有所不同, 从而影响 $\mathrm{SiO}_{3}{ }^{2-}$ 的释放行为。

基于以上假设, 本研究考虑可以通过调控硅酸 钙/高分子复合组织工程材料的孔径及硅酸钙颗粒 在复合材料中的位置来调控 $\mathrm{SiO}_{3}{ }^{2}$-释放。因此, 本研 究制备了不同孔径复合电纺丝支架, 并结合静电喷 雾技术, 构建了多种硅酸钻复合形式的电纺丝支架, 探讨了孔径及复合形式对于材料亲疏水性及离子释
放的影响, 并在此基础上考察了复合支架的不同离 子释放行为对于血管内皮细胞的影响。

\section{1 实验方法}

\section{1 实验试剂}

本研究采用的药品四水合硝酸钙 $\left(\mathrm{Ca}\left(\mathrm{NO}_{3}\right)_{2} \cdot 4 \mathrm{H}_{2} \mathrm{O}\right)$ 、九水合硅酸钠 $\left(\mathrm{Na}_{2} \mathrm{SiO}_{3} \cdot 9 \mathrm{H}_{2} \mathrm{O}\right)$ 、明 胶购于中国医药集团上海化学试剂有限公司; 聚乳 酸(PLA, 分子量 6 万)购于济南岱罡生物工程有限 公司, 聚己内酯( PCL, 分子量 8 万); 六氟异丙醇 (HFIP)购于 Sigma-Aldrich 公司。

\section{2 硅酸钲纳米线的制备与表征}

根据文献 ${ }^{[27]}$ 报道，采用水热法合成硅酸䥻纳米 线 $\left(\mathrm{Ca}_{6}\left(\mathrm{Si}_{6} \mathrm{O}_{17}\right)(\mathrm{OH})_{2}, \mathrm{CSH}\right)$ 。即, 将 $9.446 \mathrm{~g}$ 的 $\mathrm{Ca}\left(\mathrm{NO}_{3}\right)_{2} \cdot 4 \mathrm{H}_{2} \mathrm{O}$ 和 $11.368 \mathrm{~g}$ 的 $\mathrm{Na}_{2} \mathrm{SiO}_{3} \cdot 9 \mathrm{H}_{2} \mathrm{O}$ 分别溶 解于 $100 \mathrm{~mL}$ 的去离子水中, 磁力搅拌 $1 \mathrm{~h}$ 后, 将混 匀的溶液转移至水热釜中 $200{ }^{\circ} \mathrm{C}$ 反应 $24 \mathrm{~h}$ 。待水热 釜自然冷却后, 将制得的 CSH 用去离子水和乙醇分 别冲洗三次, 于 $60{ }^{\circ} \mathrm{C}$ 烘箱中烘干 $24 \mathrm{~h}$ 备用。采用 $18 \mathrm{~kW}$ 转靶 X 射线衍射仪分析 CSH 的物相组成; 使用场发射透射电子显微镜(JEM-2100F)观察纳米 线的结构。

\section{3 不同孔径电纺丝的制备}

本研究通过调节高分子电纺丝(Electrospun, ES) 溶液的浓度调节电纺丝孔径的变化。即, 按表 1 配 方将不同质量的 PLA、PCL、明胶以 35/35/30 (w/w/w) 的比例溶解于 HFIP 中, 将电纺丝溶液置于磁力摚 拌器上以 $120 \mathrm{r} / \mathrm{min}$ 的速度摚拌 $48 \mathrm{~h}$ 直至澄清, 分 别得到浓度 $(w / v)$ 为 $5 \% 、 10 \% 、 18 \% 、 22 \%$ 的电纺丝 溶液, 分别命名为 $5 \mathrm{ES} 、 10 \mathrm{ES} 、 18 \mathrm{ES} 、 22 \mathrm{ES}$ 。

\section{表 1 不同孔径电纺丝溶液的配制}

Table 1 Preparation of electrospun solution with various pore size

\begin{tabular}{ccccc}
\hline Sample & $\begin{array}{c}\text { Mass of } \\
\text { PCL/g }\end{array}$ & $\begin{array}{c}\text { Mass of } \\
\text { PLA/g }\end{array}$ & $\begin{array}{c}\text { Mass of } \\
\text { gelatin/g }\end{array}$ & $\begin{array}{c}\text { Volume of } \\
\text { HFIP/mL }\end{array}$ \\
\hline 5ES & 0.175 & 0.175 & 0.150 & 10 \\
10ES & 0.350 & 0.350 & 0.300 & 10 \\
18ES & 0.630 & 0.630 & 0.540 & 10 \\
22ES & 0.770 & 0.770 & 0.660 & 10
\end{tabular}

PCL: Polycaprolactone; PLA: Polylactic acid; HFIP: Hexafluoroisopropanol 随后各溶液分别转移到 $20 \mathrm{~mL}$ 注射器中进行静 电纺丝。由于不同浓度的电纺丝溶液的纺丝液性质 
不同, 为能制备到形态良好的电纺丝纤维, 本研究 对电纺丝的电压、流速、针头与基板的间距均进行 了相应的调节，其中各组溶液的电压、流速、针-基 板间距等纺丝参数按表 2 进行设置:

表 2 不同孔径电纺丝的制备参数

Table 2 Preparation parameters of electrospun membranes with various pore size

\begin{tabular}{cccc}
\hline Sample & $\begin{array}{c}\text { Voltage } \\
/ \mathrm{kV}\end{array}$ & $\begin{array}{c}\text { Flow rate } \\
/\left(\mathrm{mL} \cdot \mathrm{min}^{-1)}\right.\end{array}$ & $\begin{array}{c}\text { Needle-to-collec- } \\
\text { tor distance/cm }\end{array}$ \\
\hline $5 \mathrm{ES}$ & 10 & 0.02 & 8 \\
$10 \mathrm{ES}$ & 12 & 0.05 & 10 \\
$18 \mathrm{ES}$ & 15 & 0.08 & 15 \\
$22 \mathrm{ES}$ & 18 & 0.20 & 20 \\
\hline
\end{tabular}

对各组电纺丝 SEM 照片采用 ImageJ 软件测量 50 根以上的纤维, 并统计分析得到其直径和孔径 ${ }^{[28]}$, 通过测量 50 个以上由纤维围成的孔的尺寸, 并统计 分析得到其孔径。

\section{4 硅酸钙复合电纺丝支架的制备与表征}

本研究通过四种不同方式制备硅酸钙复合电纺 丝支架（图 1):（a）混纺 CSH;（b）电纺丝的同时 电喷 CSH;（c）在电纺膜表面电喷 $\mathrm{CSH}$;（d）表面 喷有 CSH 的电纺膜再进行电纺丝。四种方法制备的 电纺丝支架，分别命名为 CSH@ES、CSH\&ES、
$\mathrm{CSH} / / \mathrm{ES} 、 \mathrm{ES} / \mathrm{CSH} / \mathrm{ES}$ 。具体制备方法如下。

(a)CSH@ES: 将 $0.05 \mathrm{~g} \mathrm{CSH}$ 加入到 $10 \mathrm{~mL}$ $5 \mathrm{ES}$ 或 $22 \mathrm{ES}$ 电纺丝溶液中, 分别以表 2 所示的 $5 \mathrm{ES}$ 或 22ES 的电纺丝参数进行混纺而制得;

(b) CSH\&ES：在制作 $10 \mathrm{~mL}$ 5ES 或 22ES 电 纺丝的同时静电喷雾 $10 \mathrm{~mL}$ 含 $0.05 \mathrm{~g}$ 的 $\mathrm{CSH}$ 乙醇 溶液而制得, 其中电喷参数设定为电压 $18 \mathrm{kV}$ 及针 -基板间距 $15 \mathrm{~cm}$;

(c) $\mathrm{CSH} / / \mathrm{ES}$ ：在制作 $10 \mathrm{~mL} 5 \mathrm{ES}$ 或 22ES 电 纺丝后, 于纤维膜表面采用前述静电喷雾条件电喷 $10 \mathrm{~mL}$ 含 $0.05 \mathrm{~g}$ 的 CSH 乙醇溶液而制得;

(d) ES/CSH/ES: 在制作 $5 \mathrm{~mL} 5 \mathrm{ES}$ 或 22ES 溶 液电纺丝后, 在纤维膜上电喷 $10 \mathrm{~mL}$ 含 $0.05 \mathrm{~g}$ 的 CSH 乙醇溶液, 之后再作 $5 \mathrm{~mL} 5 \mathrm{ES}$ 或 22ES 溶液电 纺丝, 形成以 $\mathrm{CSH}$ 为夹心层的 “三明治” 纤维膜结 构。

上述制备过程中，通过控制掺入或电喷相同含 量的 CSH 以保证各组的 CSH 含量一致。

硅酸钲复合支架的接触角表征通过水接触角分 析仪(FM40 Mk 2 Easydrop)进行测试; 利用电感耦合 等离子体发射光谱仪 (ICP-OES) 检测支架在细胞培 养液中浸泡 $1 、 3 、 5 、 7 \mathrm{~d}$ 的 $\mathrm{SiO}_{3}$-释放行为。 (a)

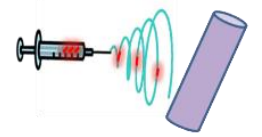

(b)

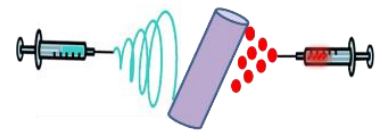

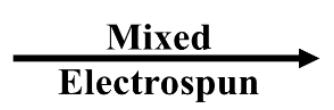

$\underset{\text { Electrospun-Electrospray }}{\stackrel{\text { Simultaneously }}{\longrightarrow}}$
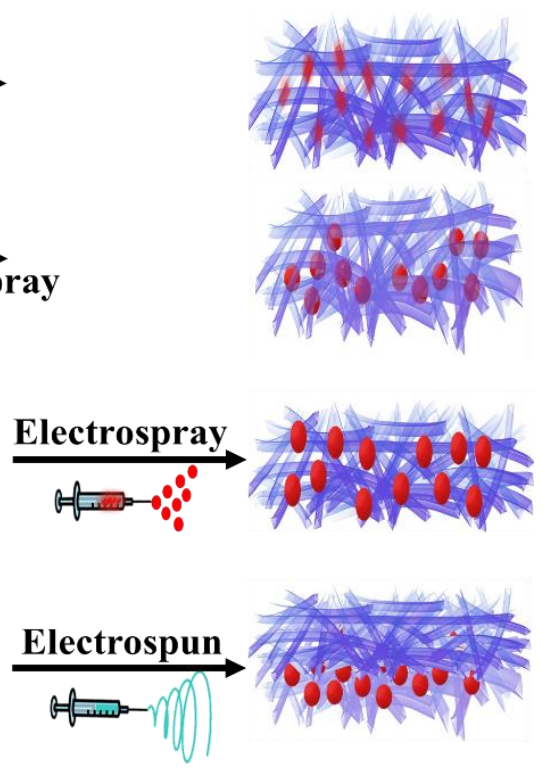

图 1 多种形式硅酸钲纳米线复合电纺丝纤维支架制备示意图

Fig.1 Schematic illustration of different preparation methods for calcium silicate composite electrospun scaffolds with different forms

(a) CSH@ES: mixed electrospun of CSH-contained blended electrospun solution; (b) CSH\&ES: simultaneously electrospraying CSH particles with elctrospun fibers; (c) CSH/ES: electrospraying CSH particles on the surface of as-spun electrospun membranes; (d) ES/CSH/ES: electrospraying CSH particles on asspun electrospun membranes, then electrospun fibers on the surface to construct an ES/CSH/ES sandwich structure

\section{5 细胞实验}

本研究中采用人脐静脉内皮细胞(HUVEC, 购
自中科院细胞库)研究硅酸钻复合电纺丝支架对细 胞的增殖影响。将 HUVEC 种于纤维支架表面, 并 培养于 $37{ }^{\circ} \mathrm{C}$ 、含 $5 \%$ 二氧化碳空气的细胞培养箱中, 
隔天更换细胞培养液。在预设时间点 $1 、 3 、 7 \mathrm{~d}$ 时 采用 Cell Counting Kit-8 (CCK8，Japan)试剂盒对细 胞活性进行检测。

为了观察 HUVEC 在材料上的粘附效果, 将培 养 $1 、 3 \mathrm{~d}$ 后的细胞样品培养液吸出, 并用 $4 \%$ 多聚 甲醛固定 $30 \mathrm{~min}$ 后, 采用苂光素异硫氧酸酯(FITC)鬼笔环肽浸泡材料上的细胞 $30 \mathrm{~min}$ 以染色细胞骨 架, 用 DAPI 浸泡 $10 \mathrm{~min}$ 以染色细胞核, 之后在激 光共聚焦显微镜下观察细胞形态。

\section{2 结果与讨论}

\section{$2.1 \mathrm{CSH}$ 的组成与形貌}

本研究通过水热法成功制备了硅酸钙纳米线。 通过 XRD 对 CSH 的物相进行了表征并与标准卡片 PDF\#23-0125 $\left(\mathrm{Ca}_{6} \mathrm{Si}_{6} \mathrm{O}_{17}(\mathrm{OH})_{2}\right)$ 比较, 结果显示 $\mathrm{CSH}$ 的主要峰位均与 $\mathrm{Ca}_{6} \mathrm{Si}_{6} \mathrm{O}_{17}(\mathrm{OH})_{2}$ 保持一致(图 2(a))。

(a)

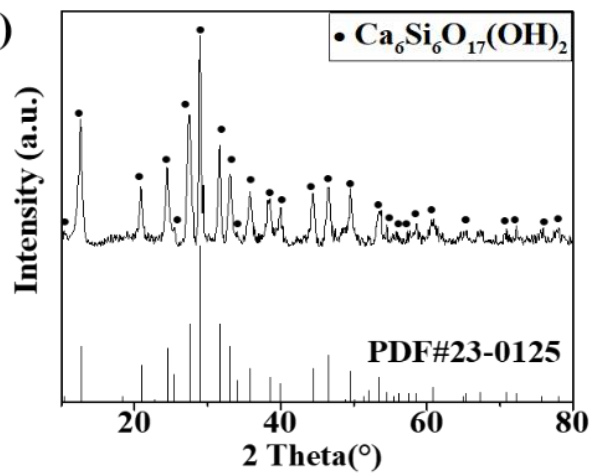

(b)

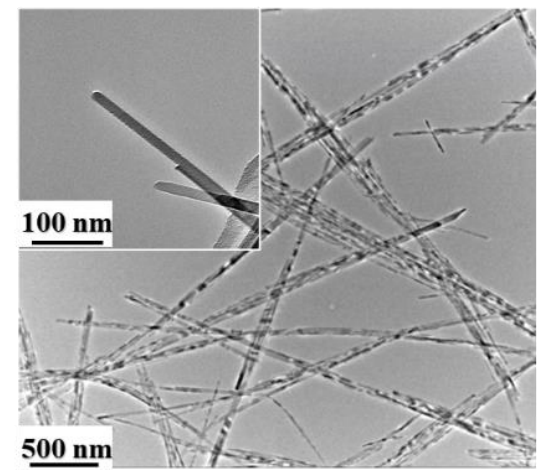

图 2 硅酸钙纳米线的（a）XRD 图谱和（b）TEM 照片

Fig.2 (a) XRD patterns and (b) TEM images of calcium silicate nanowires with inset in (b) showing magnified TEM image of $\mathrm{CSH}$

如图 2(b) TEM 照片所示, 水热法制备的 CSH 纳米线具有一维结构, 平均直径约 $20 \mathrm{~nm}$, 长度 3 7 $\mu \mathrm{m}$ 。

\section{2 不同孔径的电纺丝形貌}

在未复合硅酸钙纳米线的情况下, 通过调节电
纺丝溶液浓度与电纺丝参数，分别制备了不同结构 的电纺丝纤维。如图 3 所示, 随着电纺丝溶液浓度 的增大并相应调节电压、流速、针头与基板等电纺 丝参数后, 可看出所制得的纤维直径和相应的孔径 都逐渐增大。

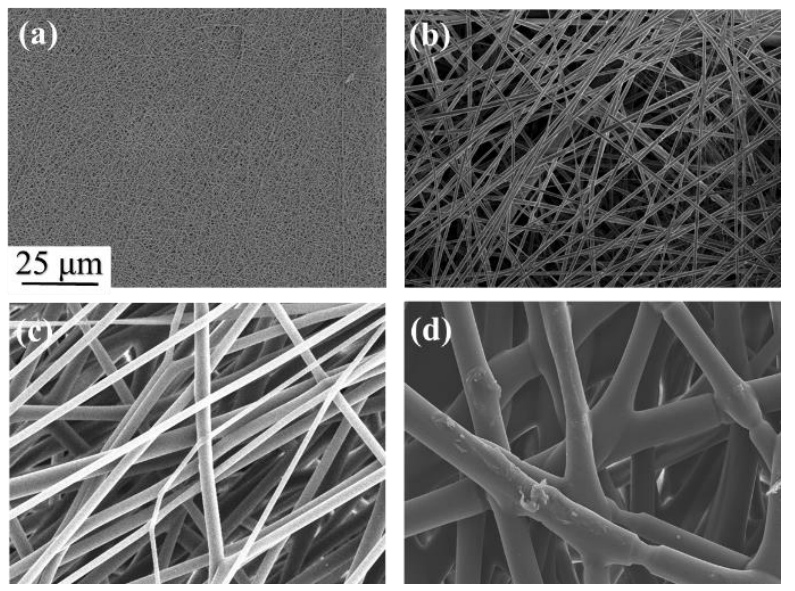

图 3 不同孔径未复合 CSH 纳米线的电纺丝纤维支架的 SEM 照片

Fig.3 SEM images of electrospun scaffolds contained no CSH nanowires with different pore sizes

(a) $5 \mathrm{ES}$; (b) 10ES; (c) $15 \mathrm{ES}$; (d) $22 \mathrm{ES}$

进一步对电纺丝作纤维直径与孔径的测定和统 计分析（表 3) 表明, 纤维直径与孔径最小的 5ES 组, 其纤维直径和孔径分别为 0.24 和 $0.68 \mu \mathrm{m}$; 纤 维直径与孔径最大的 $22 \mathrm{ES}$ 组, 其纤维直径和孔径 分别为 10.27 和 $50.46 \mu \mathrm{m}$ 。后续实验将采用孔径最 小的 $5 \mathrm{ES}$ 和孔径最大的 $22 \mathrm{ES}$ (分别命名为小孔径 组和大孔径组）探索孔径对离子释放的影响。

表 3 电纺支架的纤维直径及孔径统计结果

Table 3 Statistical results of fiber diameter and pore diameter of electrospun scaffolds

\begin{tabular}{ccc}
\hline Sample & $\begin{array}{c}\text { Fiber diameter } \\
/ \mu \mathrm{m}\end{array}$ & $\begin{array}{c}\text { Pore diameter } \\
/ \mu \mathrm{m}\end{array}$ \\
\hline 5ES & $0.24 \pm 0.05$ & $0.68 \pm 0.13$ \\
$10 \mathrm{ES}$ & $0.82 \pm 0.16$ & $3.45 \pm 0.73$ \\
$18 \mathrm{ES}$ & $4.112 \pm 0.81$ & $17.73 \pm 3.53$ \\
$22 \mathrm{ES}$ & $10.27 \pm 2.04$ & $50.46 \pm 10.87$ \\
\hline
\end{tabular}

\section{3 硅酸钙复合电纺丝支架的形貌特征及润湿} 性能

本研究通过将电纺丝溶液与 CSH 进行混纺制 备了CSH@ES, 在电纺丝纤维的同时电喷 CSH 颗 粒，制备得到了 CSH\&ES，在已纺纤维表面电喷 $\mathrm{CSH}$ 颗粒制备了 $\mathrm{CSH} / / \mathrm{ES}$ ，在表面电喷了 $\mathrm{CSH}$ 的 电纺丝表面再纺丝一层构建 CSH 的夹心 “三明治” 结构得到 $\mathrm{ES} / \mathrm{CSH} / \mathrm{ES}$ ，其形貌分别如图 4 所示。 
混纺的 CSH@ES 的电纺丝纤维表面光滑（图 $4(a, b))$, 无肉眼可见的 CSH 纳米线分布于纤维表 面, 但通过 TEM 观察可知, CSH 均匀分散于纤维 丝内部（图 4(a)插图)。由于电喷过程中，喷出的液 滴中乙醇溶剂不断挥发, CSH 纳米线发生团聚, 最 终在基底上形成 CSH 团聚体颗粒。同时电喷-电纺 的 CSH\&ES (图 4(c,d)), 颗粒均匀分布于整个电纺 丝支架体系内, $\mathrm{CSH}$ 团聚体颗粒不仅分布于支架表 面, 还大量被电纺纤维缠绕镶嵌于纤维支架中（图 4(c)插图)。而 $\mathrm{CSH} / \mathrm{ES}$ 中, 大量 $\mathrm{CSH}$ 团聚体颗粒 因静电作用均匀吸附于电纺丝纤维表面 (图 4(e,f)), 图 4（e）插图展示了 $\mathrm{CSH} / / \mathrm{ES}$ 支架表面的 CSH 团 聚体颗粒的放大照片, 可以看到颗粒由大量细小的 纳米线团聚而成，其直径在 1 2 $\mu \mathrm{m}$ 。而 “三明治” 结构的 $\mathrm{ES} / \mathrm{CSH} / \mathrm{ES}$, 因 CSH 位于夹心层位置, 表面 仅可观察到光滑的电纺丝, 无明显的 CSH 暴露 (图 $4(\mathrm{~g}, \mathrm{~h}))$ 。

此外, 以小孔径电纺丝为例, 掺入 $\mathrm{CSH}$ 纳米线 的电纺丝 CSH@ES 的纤维直径为 $(0.26 \pm 0.03) \mu \mathrm{m}$, 孔径为 $(0.74 \pm 0.18) \mu \mathrm{m}$, 这与未复合 $\mathrm{CSH}$ 的 $\mathrm{ES}$ 电纺丝的直径及孔径（表 3 的 5ES 组）并无显著性 差异。电喷 $\mathrm{CSH}$ 颗粒的 CSH\&ES、 CSH//ES 和 $\mathrm{ES} / \mathrm{CSH} / \mathrm{ES}$ 电纺丝支架, 经统计分析直径分别为 $(0.28 \pm 0.09) \mu \mathrm{m} 、(0.25 \pm 0.05) \mu \mathrm{m} 、(0.23 \pm 0.03)$ $\mu \mathrm{m}$, 孔径分别为 $(0.79 \pm 0.15) \mu \mathrm{m} 、(0.70 \pm 0.09)$ $\mu \mathrm{m} 、(0.64 \pm 0.05) \mu \mathrm{m}$ 、与 $\mathrm{ES}$ 组也并无显著性差异。 以上结果说明, 引入 $\mathrm{CSH}$ 并未显著改变电纺丝支架 的纤维直径及孔径因素。
Small pore

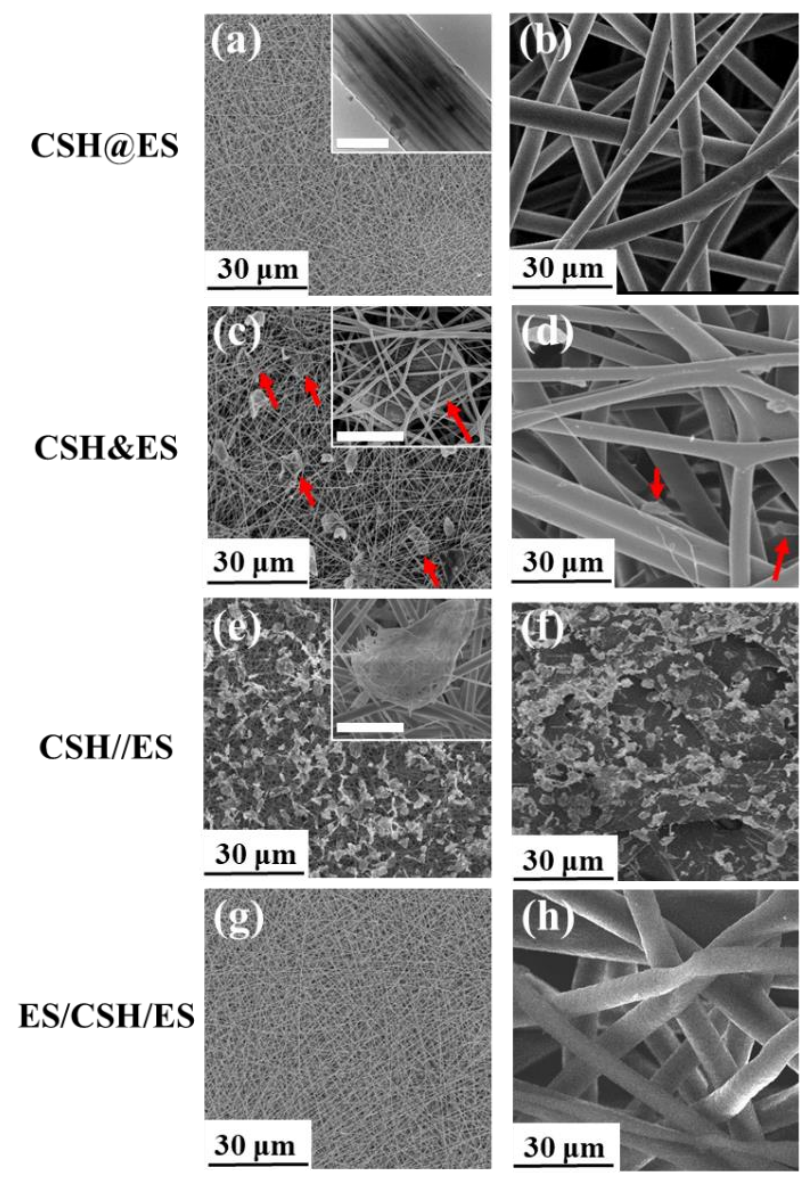

图 4 多种复合形式的硅酸钻纳米线不同孔径电纺丝支架的 SEM 照片及 CSH 颗粒在支架内的分布

Fig. 4 SEM images of various forms of calcium silicate composite electrospun scaffolds with different pore sizes and CSH particle distributions in the scaffolds

(a, b) CSH@ES; (c, d) CSH\&ES; (e, f) CSH//ES; (g, h) ES/CS/ES; (a, c, e, g) Small pore size; (b, d, f, h) Large pore size. The inset in (a) shows CSH nanowires being uniformly distributed inside the fibers, scale bar $=200 \mathrm{~nm}$. The inset in (c) reveals agglomerated CSH particles being embedded in the scaffolds with red arrows indicating the CSH particles embedded in scaffolds, scale bar $=4 \mu \mathrm{m}$. The inset in (e) shows the CSH nanowires formed agglomerates on the surface of the scaffolds. Scale bar $=2 \mu \mathrm{m}$ 
(a)

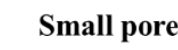

Large pore

ES
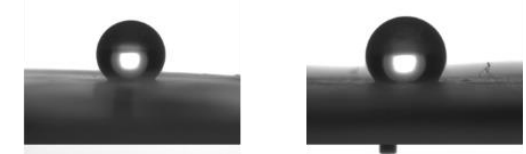

CSH@ES

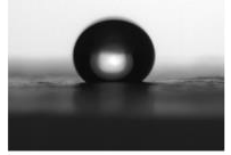

CSH\&ES
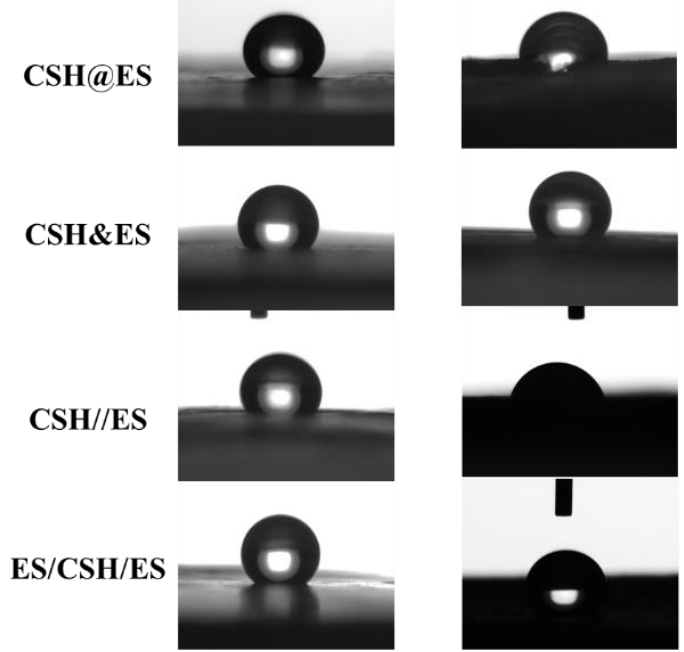

(b)

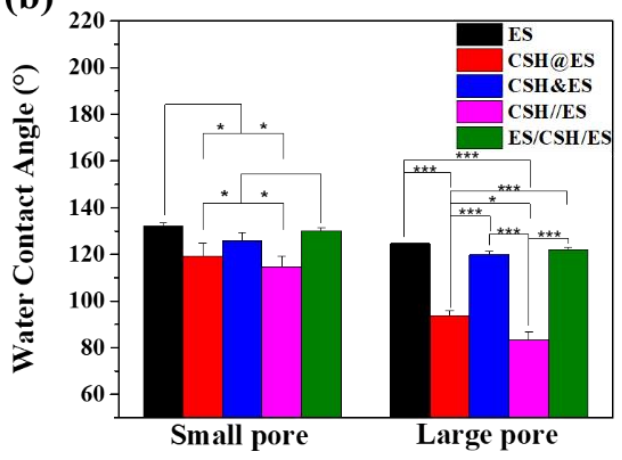

图 5 不同孔径的复合硅酸钻纳米线电纺丝支架的接触角

Fig. 5 Water contact angle measurements of various forms of calcium silicate composite electrospun scaffolds with different pore sizes

(a) Photographs of water contact angle on electrospun scaffolds; (b) Statistical results of water contact angle. $* p<0.05, * * p<0.01, * * * p<$ 0.001

材料的亲疏水性可影响环境中的液体扩散到材 料内部的速率, 继而影响后续释放过程 ${ }^{[23,29]}$ 。因此, 本研究中对各复合膜表面的亲疏水性能进行了测定。 如图 5 所示, 无论小孔径支架还是大孔径支架, 亲 水性相较于对照组 $\mathrm{ES}$ 均有所改善, $\mathrm{CSH} / \mathrm{ES}$ 最为 显著, CSH@ES 其次, CSH\&ES 略有改善, $\mathrm{ES} / \mathrm{CSH} / \mathrm{ES}$ 则基本没有改善。该结果与前述形貌表 征基本一致, $\mathrm{CSH} @ \mathrm{ES}$ 因 $\mathrm{CSH}$ 纳米线均匀分部在 每根纤维内部, 因此对疏水性电纺丝纤维的亲水性 有所提升; 而 CSH\&ES 表面零星分布有 CSH 颗粒, 亲水性略有改善; CSH//ES 表面均匀分布有大量 $\mathrm{CSH}$ 颗粒，所以其亲水性提升最为显著; 而 $\mathrm{ES} / \mathrm{CSH} / \mathrm{ES}$ 表面无任何 CSH 存在, 亲水性与对照样
品基本相同。由于 $\mathrm{CSH}$ 分布在电纺丝纤维表面, 因 此可以推测, 是 CSH 导致了固液界面由 Cassie 到 Wenzel 状态的转变 ${ }^{[30]}$, 从而导致了上述亲水性的变 化。
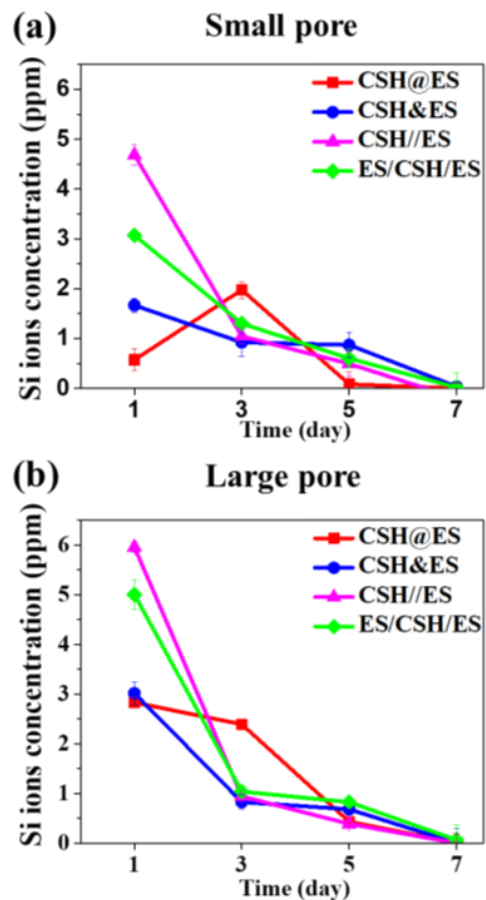

图 6 复合方式对硅酸钙纳米线电纺丝支架释放 $\mathrm{SiO}_{3}{ }^{2-}$ 行为 的影响

Fig. 6 Effects of the different composite forms on release behavior of $\mathrm{SiO}_{3}{ }^{2-}$ in calcium silicate nanowires in composite electrospun scaffolds

(a) Scaffolds with small pore size; (b) Scaffolds with large pore size

\section{4 硅酸钲复合电纺丝支架的离子释放行为}

复合支架中释放的生物活性 $\mathrm{SiO}_{3}{ }^{2-}$ 浓度对于细 胞的增殖等有重要影响。因此, 本研究系统测试了 小孔径和大孔径支架中，不同复合方式的硅酸钻离 子释放行为, 结果如图 6 所示。在复合 CSH 方式相 同的情况下，对比大孔径及小孔径支架，二者的离 子释放曲线趋势基本相同, 但大孔径的离子释放曲 线更为陡峭, 突释的现象更为明显, 这可能与大孔 径带来更快的扩散速度有关 ${ }^{[22-24]}$ 。

但无论在何种孔径的支架中, CSH//ES 及 $\mathrm{ES} / \mathrm{CSH} / \mathrm{ES}$ 在早期的突释效应明显, 而 CSH@ES 及 CSH\&ES 有一定的缓释作用。值得一提的是, 四 组样品中 $\mathrm{CSH} / / \mathrm{ES}$ 的离子释放最快, CSH\&ES 组释 放曲线相对较为平缓; 小孔径 CSH\&ES 支架中, 其

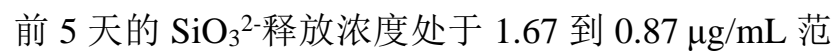
围内, 较长时间处于理论的 $0.79 \sim 1.8 \mu \mathrm{g} / \mathrm{mL}$ 有效 $\mathrm{SiO}_{3}{ }^{2-}$ 浓度范围 ${ }^{[22]}$ 内; 更有价值的是, $\mathrm{CSH} @ \mathrm{ES}$ 组 
在第 3 天的离子浓度出现了明显的拐点, 特别是小 孔径 CSH@ES 组尤为明显, 其释放量从第 1 天的 $0.57 \mu \mathrm{g} / \mathrm{mL}$ 提升到第 3 天的 $1.97 \mu \mathrm{g} / \mathrm{mL}$ ，而后又逐 渐下降。

根据文献 ${ }^{[31]}$ 报道，电纺丝纤维支架中的药物释 放主要机理为: (1)扩散, 液体因浓度梯度扩散进入 或离开高分子体系内部; (2)松弛, 高分子链段松弛 从而使液体进入体系内部; (3)溶出/降解, 在液体环 境作用下物质溶出/降解。基于这一理论并根据本研 究中 CSH 的分布位置、亲水性、离子释放的结果, 本研究认为, 硅酸钙复合电纺丝体系的降解机理可 用图 7 说明, 解释了 CSH@ES 和 CSH\&ES 组的离 子缓释作用。

对于 CSH@ES 组及 CSH\&ES 组（图 7(a)）, 由于 $\mathrm{CSH}$ 纳米颗粒位于单根纤维内部或者被纤维 丝紧密包裹, 高分子的疏水作用 ${ }^{[26]}$ 使得环境中的水 分子难以快速扩散到高分子内部, 因此 CSH 降解需 要经历高分子链段的松弛或部分降解过程后, 水分 子方可进入支架内部与 CSH 接触, 从而降解释放出 离子。特别地, 由于 PCL、PLA 等疏水高分子降解
缓慢 ${ }^{[32]}, \mathrm{CSH} @ \mathrm{ES}$ 小孔径支架的第 1 天释放的离子 浓度反而较低, 待高分子部分降解后, 离子释放才 逐渐增多; 而在大孔径支架中，由于表面亲水性较 强, 孔径越大水分子扩散速度越快 ${ }^{[33]}$, 虽然观测到 离子释放的拐点，但第 1 天释放的离子浓度更高。

对于 $\mathrm{CSH} / / \mathrm{ES}$, 环境中的水分子可以快速扩散 到材料表面使 CSH 发生降解并产生离子突释; 如图 7(b)所示, 对于 ES/CSH/ES 组, 本研究推测虽然 “夹 心层” 结构的上表面疏水性较强, 但一旦液体渗透 疏水的上表面后便会与 $\mathrm{CSH}$ “夹心层” 直接接触, 导致陶瓷颗粒的直接释放，因此其释放速度较 $\mathrm{CSH}$ 被包裹在高分子内部的各组更快。但相比之下，环 境中的液体扩散到 $\mathrm{ES} / \mathrm{CSH} / \mathrm{ES}$ 夹心层陶瓷处的扩 散距离较 $\mathrm{CSH} / / \mathrm{ES}$ 表面接触更长, 且一定程度受上 下两层高分子的阻挡作用, 因此其突释速度稍慢于 $\mathrm{CSH} / \mathrm{ES}$ 组。

综上, 与 $\mathrm{CSH} / / \mathrm{ES}$ 和 $\mathrm{ES} / \mathrm{CSH} / \mathrm{ES}$ 相比, 由于 CSH@ES 及 CSH//ES 分别需要经历高分子部分降 解及高分子链段松弛的过程, 因此这两组硅酸钙复 合材料的离子释放具有一定的缓释效应。 (a)

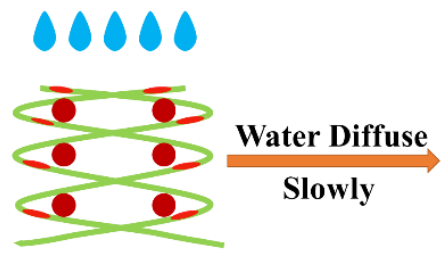

(b)
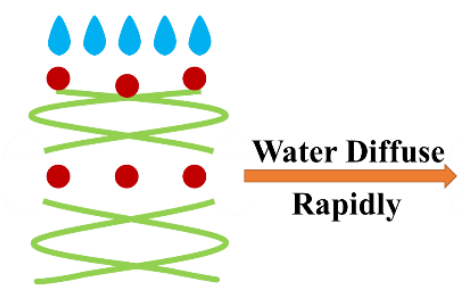

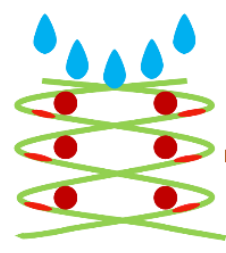

Polymer Erode Polymer Relax

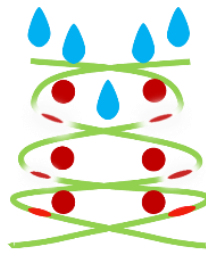

Ions Release

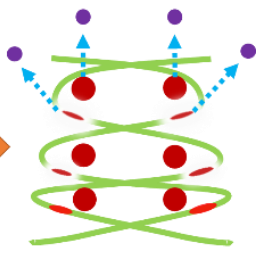

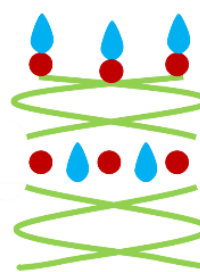
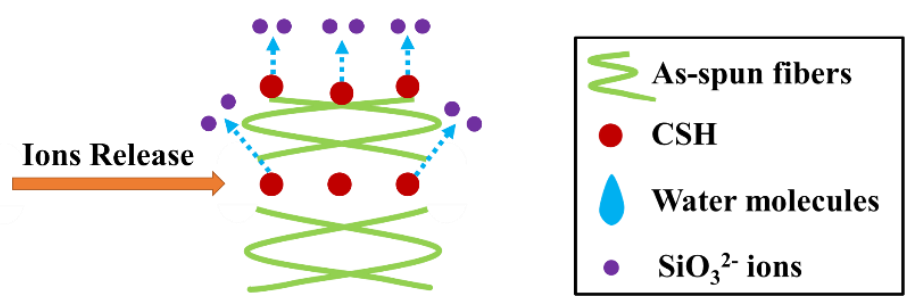

图 7 硅酸钙复合电纺丝纤维支架离子释放过程示意图

Fig. 7 Schematic illustrations of ions release behaviors from calcium silicate composite in electrospun scaffolds (a) Ions controlled release of CSH@ES and CHS\&ES groups; (b) Ions burst release process of CSH//ES and ES/CSH/ES groups

2.5 HUVEC 在硅酸钙复合电纺丝支架表面的细 胞增殖及细胞形态

考虑到大孔径支架造成的扩散速度较快，各组 离子释放的突释效应均较为明显, 因此本研究优选 了小孔径各复合电纺支架进行比较，考察不同离子 释放模式对于内皮细胞增殖的影响。结果如图 8 所 示, 培养 $7 \mathrm{~d}$ 时, 相较于不含 CSH 的 ES 组, CSH 复合电纺丝支架各组均一定程度上促进了内皮细胞 的增殖, 说明复合纤维膜释放的 $\mathrm{SiO}_{3}{ }^{2-}$-促进了细胞 增殖。进一步比较发现, 培养 $3 \mathrm{~d}$ 时, 具有离子缓
释效果的 CSH@ES 与 CSH\&ES 促进了细胞增殖, 而突释的 $\mathrm{CSH} / / \mathrm{ES}$ 及 $\mathrm{ES} / \mathrm{CSH} / \mathrm{ES}$ 组则无促进增殖 效果; 培养 $7 \mathrm{~d}$ 时, 缓释的两组与突释的两组对比 也显示, 缓释组的促进增殖效果更明显。这可能是 早期突释现象造成了离子浓度过高不利于细胞增殖, 而缓释组的 $\mathrm{SiO}_{3}$ 2-浓度能够更有效地促进细胞增殖。

事实上, 电纺丝的纤维直径、孔径、孔隙率和 力学性能等均有可能影响细胞的增殖行为, 但本研 究制备的 ES、CSH@ES、CSH\&ES、CSH//ES 和 $\mathrm{ES} / \mathrm{CSH} / \mathrm{ES}$ 各组支架间, 电纺丝的直径和孔径并无 
显著性差异。而文献 ${ }^{[34]}$ 报道，孔隙率由电纺丝的直 径和孔径决定，因此孔隙率也无显著性差异。在力 学性能方面, 根据前期研究报道, 电纺丝中掺入 $1 \% \mathrm{CSH}$ 纳米线后, 各力学参数以及应力-应变曲线 无明显变化 ${ }^{[19]}$, 掺入 $10 \%$ 以上 $\mathrm{CSH}$ 纳米线才能显 著提高电纺丝的拉伸强度 ${ }^{[35]}$, 而本研究中加入的 $\mathrm{CSH}$ 含量仅为 $0.5 \%$, 因此可以认为加入 $\mathrm{CSH}$ 对于 材料的力学性能影响较小, 对细胞的增殖促进作用 可以忽略。综上，可以合理地推定，细胞增殖的差 异主要是由于材料中的 $\mathrm{SiO}_{3}{ }^{2-}$ 生物活性离子释放所 导致的。

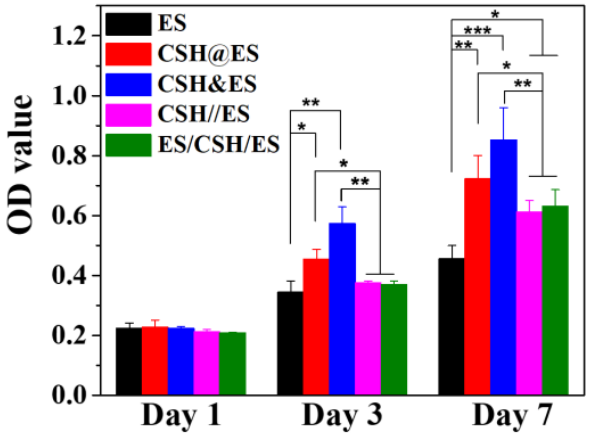

图 8 人脐静脉内皮细胞在小孔径不同复合方式电纺丝纤维 支架上培养 1、3、7 d 时的增殖

Fig. 8 Proliferation of HUVECs after being cultured on various composite electrospun scaffolds with small pore. ${ }^{*} p<0.05$, ** $p<0.01$, ***p $<0.001)$

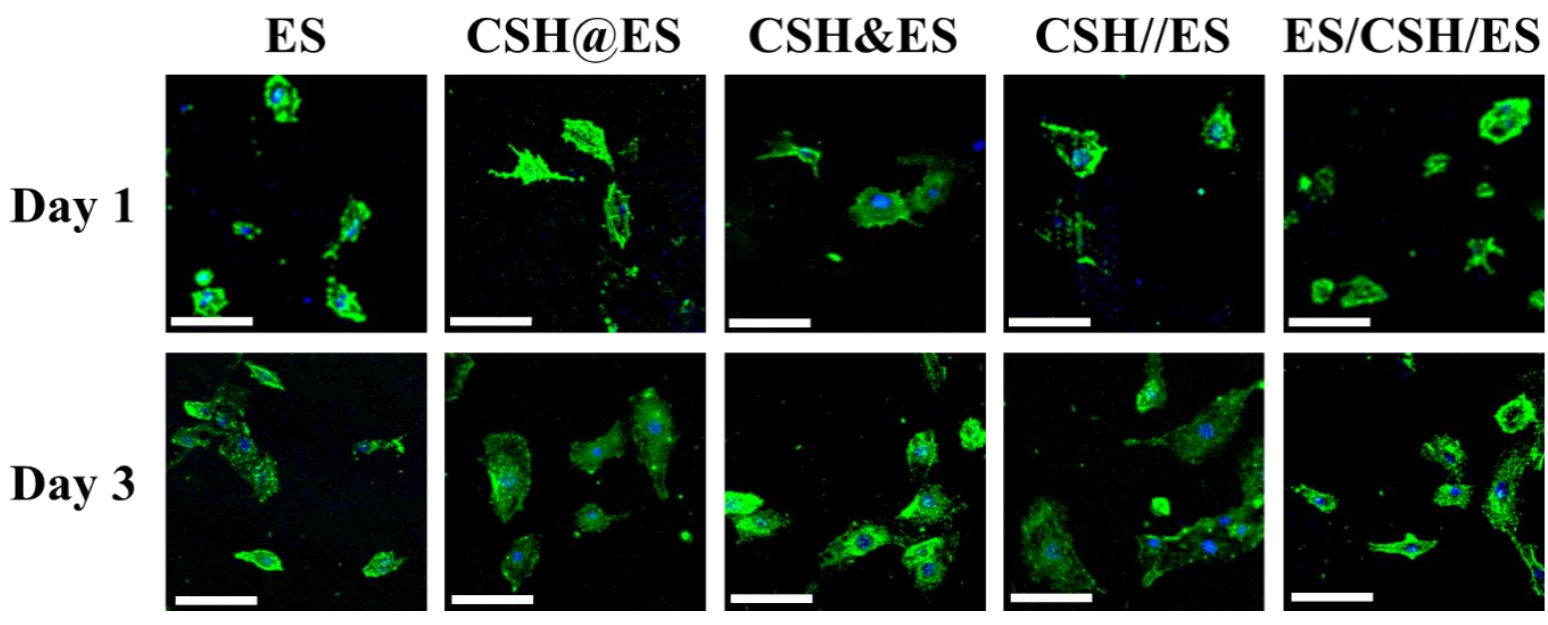

图 9 人脐静脉内皮细胞在小孔径不同方式复合电纺丝纤维支架上培养 $1 、 3 \mathrm{~d}$ 时的粘附形态

Fig. 9 HUVEC morphology after being cultured on various composite electrospun scaffolds with small pore for 1 and $3 \mathrm{~d}$ Scale bars $=100 \mu \mathrm{m}$

将 HUVEC 分别在小孔径各复合形式电纺支架 表面培养 1 和 $3 \mathrm{~d}$ 时, 观察材料表面的细胞粘附状 态（图 9），可以发现在培养 $1 \mathrm{~d}$ 时, ES 组和 $\mathrm{ES} / \mathrm{CSH} / \mathrm{ES}$ 组表面的细胞铺展较差, 大多呈球形, 而 CSH@ES、CSH\&ES、CSH//ES 组的细胞呈现少 量伪足; 在培养 $3 \mathrm{~d}$ 时, 各电纺丝组表面的细胞均 较 $1 \mathrm{~d}$ 时铺展明显, 特别是 CSH@ES、CSH\&ES、 $\mathrm{CSH} / / \mathrm{ES}$ 组的效果更好。这可能是由于 $\mathrm{ES}$ 及 $\mathrm{ES} / \mathrm{CSH} / \mathrm{ES}$ 表面的疏水性较强, 而 $\mathrm{CSH}$ 的引入导致 了 CSH@ES、CSH\&ES、CSH//ES 组电纺丝表面的 亲水性在一定程度上的改善, 从而促进了细胞的粘 附。

\section{3 结论}

本研究采用硅酸钻高分子复合电纺丝膜材料研 究了复合材料释放 $\mathrm{SiO}_{3}{ }^{2}$ 规律及其细胞生物学效应, 可以得出如下结论:

1) 通过调节电纺丝溶液浓度获得了不同孔径大小 的电纺丝支架; 通过静电纺丝与静电喷雾技术 的不同组合方式，制备得到了具有不同分布特 点的硅酸钙纳米颗粒/高分子复合电纺丝材料;

2）在相同陶瓷颗粒分布的情况下，电纺丝支架孔 径越小，离子释放越缓慢;

3）电纺丝支架在具有相同孔径的情况下，硅酸钻 纳米线分布于纤维丝内部或被纤维丝紧密缠绕 在支架内部的复合电纺丝材料, 由于疏水高分 子包裹效应导致离子释放缓慢, 具有缓释特性; 
4) 具有离子缓释作用的小孔径混纺及同时电纺-电 喷陶瓷/高分子复合电纺丝材料可有效促进内皮 细胞的增殖。

5) 本研究通过调节孔径和 CSH 的复合方式, 发现 小孔径及混纺或同时电喷-电纺的复合形式对于 离子缓释效果最佳, 验证了其在促进细胞增殖 方面的优势, 有望为新型创伤敷料的开发提供 参考。

但是，体内的液体动态流动环境与体外模拟的 液体环境存在较大差别, 复合支架在体内的最佳促 修复离子释放浓度也与体外存在一定差异，本研究 得出的上述组织工程复合支架材料的规律还有待动 物实验的验证。

\section{参考文献}

[1] XIE X, CHEN Y, WANG X, et al. Electrospinning nanofiber scaffolds for soft and hard tissue regeneration. Journal of Materials Science \& Technology, 2020, 59:243-261.

[2] ZHANG Y, DOU L, MA N, et al. Biomedical applications of electrospun nanofibers. Surface Review and Letters, 2020, 27(11): 126.

[3] NIEMCZYK-SOCZYNSKA B, GRADYS A, SAJKIEWICZ P. Hydrophilic surface functionalization of electrospun nanofibrous scaffolds in tissue engineering. Polymers, 2020, 12(11): 2636.

[4] XU F, FAN Y. Electrostatic self-assemble modified electrospun poly-L-lactic acid/poly-vinylpyrrolidone composite polymer and its potential applications in small-diameter artificial blood vessels. Journal of Biomedical Nanotechnology, 2020, 16(1): 101-110.

[5] AWAD N K, NIU H, ALI U, et al. Electrospun fibrous scaffolds for small-diameter blood vessels: a review. Membranes, 2018, 8(1): 15 .

[6] HAN J, WU Q, XIA Y, et al. Cell alignment induced by anisotropic electrospun fibrous scaffolds alone has limited effect on cardiomyocyte maturation. Stem Cell Research, 2016, 16(3): 740-750.

[7] SUH T C, AMANAH A Y, GLUCK J M. Electrospun scaffolds and induced pluripotent stem cell-derived cardiomyocytes for cardiac tissue engineering applications. Bioengineering, 2020, 7(3): 105.

[8] AMINI S, SAUDI A, AMIRPOUR N, et al. Application of electrospun polycaprolactone fibers embedding lignin nanoparticle for peripheral nerve regeneration: in vitro and in vivo study. International Journal of Biological Macromolecules, 2020, 159: 154-173.

[9] ZHA F, CHEN W, HAO L, et al. Electrospun cellulose-based conductive polymer nanofibrous mats: composite scaffolds and their influence on cell behavior with electrical stimulation for nerve tissue engineering. Soft Matter, 2020, 16(28): 6591-6598.

[10] BAO F, PEI G, WU Z, et al. Bioactive self-pumping composite wound dressings with micropore array modified Janus membrane for enhanced diabetic wound healing. Advanced Functional Materials, 2020, 30(49): 1-11.

[11] REN X Z, HAN Y M, WANG J, et al. An aligned porous electrospun fibrous membrane with controlled drug delivery - an efficient strategy to accelerate diabetic wound healing with improved angiogenesis. Acta Biomaterialia, 2018, 70: 140-153.

[12] WANG X C, Lv F, Li T, et al. Electrospun micropatterned nanocomposites incorporated with $\mathrm{Cu}_{2} \mathrm{~S}$ nanoflowers for skin tumor therapy and wound healing. ACS Nano, 2017, 11(11): 11337-11349.

[13] XIA L, YIN Z, MAO L, et al. Akermanite bioceramics promote osteogenesis, angiogenesis and suppress osteoclastogenesis for osteoporotic bone regeneration. Scientific Reports, 2016, 6: 22005.

[14] ZHANG Y, HUANG C, CHANG J. Ca-doped mesoporous $\mathrm{SiO} 2 /$ dental resin composites with enhanced mechanical properties, bioactivity and antibacterial properties. Journal of Materials Chemistry B, 2018, 6(3): 477-486.

[15] LI J, ZHAI D, LV F, et al. Preparation of copper-containing bioactive glass/eggshell membrane nanocomposites for improving angi- ogenesis, antibacterial activity and wound healing. Acta Biomaterialia, 2016, 36: 254-66.

[16] WANG X, GAO L, HAN Y, et al. Silicon-enhanced adipogenesis and angiogenesis for vascularized adipose tissue engineering. Advanced Science, 2018, 5(11): 1800776.

[17] YU Q, HAN Y, WANG X, et al. Copper silicate hollow microspheres-incorporated scaffolds for chemo-photothermal therapy of melanoma and tissue healing. ACS Nano, 2018, 12(3): 2695-2707.

[18] ZHANG Z, DAI Q, ZHANG Y, et al. Design of a multifunctional biomaterial inspired by ancient Chinese medicine for hair regeneration in burned skin. ACS Applied Materials \& Interfaces, 2020, 12(11): 12489-12499.

[19] WANG X, WANG L, WU Q, et al. Chitosan/calcium silicate cardiac patch stimulates cardiomyocyte activity and myocardial performance after infarction by synergistic effect of bioactive ions and aligned nanostructure. ACS Applied Materials \& Interfaces, 2019, 11(1): 1449-1468.

[20] LI J Y, LV F, XU H, et al. A patterned nanocomposite membrane for high-efficiency healing of diabetic wound. Journal of Materials Chemistry B, 2017, 5(10): 1926-1934.

[21] YU Q, CHANG J, WU C. Silicate bioceramics: from soft tissue regeneration to tumor therapy. Journal of Materials Chemistry B, 2019, 7(36): 5449-5460.

[22] LI H, CHANG J. Stimulation of proangiogenesis by calcium silicate bioactive ceramic. Acta Biomaterialia, 2013, 9(2): 5379-5389.

[23] LIU Z, JU X J, HUANG Y H, et al. Diffusional permeability characteristics of positively $\mathrm{K}^{+}$-responsive membranes caused by spontaneously changing membrane pore size and surface wettability. Journal of Membrane Science, 2016, 497: 328-338.

[24] SUN H, CHEN J, HAN X, et al. Multi-responsive hydrogels with UCST- and LCST-induced shrinking and controlled release behaviors of rhodamine B. Materials Science \& Engineering C-Materials for Biological Applications, 2018, 82: 284-290.

[25] ZHU W, WAN L, ZHANG C, et al. Exploitation of 3D face-centered cubic mesoporous silica as a carrier for a poorly water soluble drug: influence of pore size on release rate. Materials Science \& Engineering C-Materials for Biological Applications, 2014, 34: 78-85.

[26] DANTAS M J L, SANTOS B F F D, TAVARES A A, et al. The impact of the ionic cross-linking mode on the physical and in vitro dexamethasone release properties of chitosan/hydroxyapatite beads. Molecules, 2019, 24(24): 4510.

[27] LIN K L, CHANG J, CHEN G F, et al. A simple method to synthesize single-crystalline $\beta$-wollastonite nanowires. Journal of Crystal Growth, 2006, 300(2): 267-271.

[28] NISHA U, MERLINE C, LAKSHMINARAYANAN R, et al. Tunable release of combined contraceptive steroids from core-shell gelatin/PCL fibers. Fibers and Polymers, 2020, 21(9): 1906-1916.

[29] YOHE S T, HERRERA V L M, COLSON Y L, et al. 3D super hydrophobic electrospun meshes as reinforcement materials for sustained local drug delivery against colorectal cancer cells. Journal of Control Release, 2012, 162(1): 92-101.

[30] EMAMI B, TAFRESHI H V, GAD-EL-HAK M, et al. Effect of fiber orientation on shape and stability of air-water interface on submerged super hydrophobic electrospun thin coatings. Journal of Applied Physics, 2012, 111(6): 064325.

[31] MUNJ H R, LANNUTTI J J, TOMASKO D L. Understanding drug release from PCL/gelatin electrospun blends. Journal of Biomaterials Applications, 2017, 31(6): 933-949.

[32] IMMICH A P S, ARIAS M L, CARRERAS N, et al. Drug delivery systems using sandwich configurations of electrospun poly(lactic acid) nanofiber membranes and ibuprofen. Materials Science \& Engineering C-Materials for Biological Applications, 2013, 33(7): 4002-4008.

[33] BHARADWAZ A, JAYASURIYA A. Recent trends in the application of widely used natural and synthetic polymer nanocomposites in bone tissue regeneration. Materials Science \& Engineering $C$ Materials for Biological Applications, 2020, 110: 110698.

[34] RNJAK K J, WISE G S, LI Z, et al. Tailoring the porosity and pore size of electrospun synthetic human elastin scaffolds for dermal tissue engineering. Biomaterials, 2011, 32(28): 6729-6736.

[35] DOU Y D, WU C T, CHANG J. Preparation, mechanical property and cytocompatibility of poly(l-lactic acid)/calcium silicate nanocomposites with controllable distribution of calcium silicate nanowires. Acta Biomaterialia, 2012, 8(11): 4139-4150. 
